\title{
Discordance between different bioinformatic methods for identifying resistance genes from short-read genomic data, with a focus on Escherichia coli
}

\subsection{Author names}

Timothy J Davies ${ }^{a, b}$, Jeremy Swan ${ }^{a, b}$, Anna E Sheppard ${ }^{a, b}$, Hayleah Pickford $^{a, b}$, Samuel Lipworth ${ }^{a, b}$, Manal AbuOun ${ }^{c}$, Matthew Ellington ${ }^{b,}$, Philip W Fowler $^{a}$,

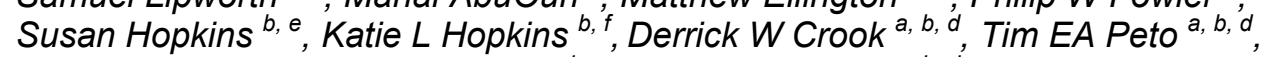
Muna F Anjum ${ }^{c}$, A Sarah Walker ${ }^{a, b}\left({ }^{*}\right)$, Nicole Stoesser ${ }^{a, b, d}\left({ }^{*}\right)$.

${ }^{*}$ contribution considered equal

\subsection{Affiliation}

a) Nuffield Department of Medicine, Oxford University, Oxford, United Kingdom

b) National Institute for Health Research (NIHR) Health Protection Research Unit on Healthcare Associated Infections and Antimicrobial Resistance at University of Oxford, UK

c) Bacteriology, Animal and Plant Health Agency, Surrey UK

d) Oxford University Hospitals NHS Foundation Trust, Oxford, UK

e) Antimicrobial Resistance and Healthcare Associated Infections (AMRHAI) Division, UK Health Security Agency, London, UK

f) HCAl, Fungal, AMR, AMU and Sepsis Division, UK Health Security Agency, London, UK

\subsection{Corresponding author}

DrTimothy Davies, timothy.davies@ndm.ox.ac.uk

Alternate corresponding author:

DrNicole Stoesser, nicole.stoesser@ndm.ox.ac.uk

\subsection{Keyword}

Antimicrobial resistance genotyping, genomics, Escherichia coli, resistance prediction

\subsection{Repositories:}

Sequencing data are available at the following NCBI BioProject accession number: PRJNA540750.

\section{Abstract}

Several bioinformatics genotyping algorithms are now commonly used to characterise antimicrobial resistance (AMR) gene profiles in whole genome sequencing (WGS) data, with a view to understanding AMR epidemiology and developing resistance prediction workflows using WGS in clinical settings. Accurately evaluating AMR in Enterobacterales, particularly Escherichia coli, is of major importance, because this is a common pathogen. However, robust comparisons of different genotyping approaches on relevant simulated and large real-life WGS datasets are lacking. Here, we used both simulated datasets and a large set of real 
44 E. coli WGS data ( $\mathrm{n}=1818$ isolates) to systematically investigate genotyping methods

45 in greater detail.

46

47

48

49

50

51

52

53

54

55

56

57

58

59

60

61

62

63

64

65

66

67

68

Simulated constructs and real sequences were processed using four different bioinformatic programs (ABRicate, ARIBA, KmerResistance, and SRST2, run with the ResFinder database) and their outputs compared. For simulations tests where 3,092 AMR gene variants were inserted into random sequence constructs,

KmerResistance was correct for all 3,092 simulations, ABRicate for 3,082 (99.7\%), ARIBA for 2,927 (94.7\%) and SRST2 for 2,120 (68.6\%). For simulations tests where two closely related gene variants were inserted into random sequence constructs, ABRicate identified the correct alleles in 11,382/46,279 (25\%) of simulations, ARIBA in 2494/46,279 (5\%), SRST in 2539/46,279 (5\%) and KmerResistance in $38,826 / 46,279(84 \%)$. In real data, across all methods, $1392 / 1818(76 \%)$ isolates had discrepant allele calls for at least one gene.

Our evaluations revealed poor performance in scenarios that would be expected to be challenging (e.g. identification of AMR genes at $<10 x$ coverage, discriminating between closely related AMR gene sequences), but also identified systematic sequence classification (i.e. naming) errors even in straightforward circumstances, which contributed to $1081 / 3092(35 \%)$ errors in our most simple simulations and at least 2530/4321 (59\%) discrepancies in real data. Further, many of the remaining discrepancies were likely "artefactual" with reporting cut-off differences accounting for at least 1430/4321 (33\%) discrepants. Comparing outputs generated by running multiple algorithms on the same dataset can help identify and resolve these artefacts, but ideally new and more robust genotyping algorithms are needed.

\section{Impact statement}

Whole-genome sequencing is widely used for studying the epidemiology of antimicrobial resistance (AMR) genes in bacteria; however, there is some concern that outputs are highly dependent on the bioinformatics methods used. This work evaluates these concerns in detail by comparing four different, commonly used AMR gene typing methods using large simulated and real datasets. The results highlight performance issues for most methods in at least one of several simulated and reallife scenarios. However most discrepancies between methods were due to differential labelling of the same sequences related to the assumptions made regarding the underlying structure of the reference resistance gene database (i.e. that resistance genes can be easily classified in well-defined groups). This study represents a major advance in quantifying and evaluating the nature of discrepancies between outputs of different AMR typing algorithms, with relevance for historic and future work using these algorithms. Some of the discrepancies can be resolved by choosing methods with fewer assumptions about the reference AMR gene database and manually resolving outputs generated using multiple programs. However, ideally new and better methods are needed. 


\section{Introduction}

Whole genome sequencing (WGS) has become a major tool for characterising the epidemiology of bacterial antimicrobial resistance (AMR) genes, representing a potentially highly discriminatory, non-targeted approach with significant advantages over other more targeted molecular techniques(1). In addition, WGS-based antibiotic susceptibility prediction has been successfully implemented as part of diagnostic and treatment workflows for Mycobacterium tuberculosis(2). Accurate WGS-based profiling of complete AMR gene content and prediction of susceptibility phenotypes would represent an attractive option for other commonly encountered clinical bacterial pathogens, such as Enterobacterales, including Escherichia coli.

Several key components are required for WGS-based AMR genotyping and predictions of susceptibility phenotype, including a robust AMR gene reference catalogue linking each genetic mechanism/sequence with a given phenotype, and accurate AMR gene identification and classification algorithms. Several catalogues and bioinformatics algorithms are now available(3-9), but only limited comparative evaluation of their outputs has been undertaken. The genetic mechanisms underpinning AMR in Enterobacterales and some other bacteria (e.g. Pseudomonas aeruginosa) are much more complex than those in M. tuberculosis, and whilst some studies suggest that WGS-based genotyping holds promise for AMR gene characterisation and the prediction of antimicrobial susceptibility for several different Enterobacterales species(10-12), the limited reproducibility and reliability of such methods in a blinded, head-to-head analysis across nine bioinformatics teams has been recently highlighted(13). However, this study was small $(n=10$ sequencing datasets, $n=7$ isolates), encountered a limited set of typing discrepancies, and used highly selected samples, meaning the impact of these issues on larger, real-world datasets remains unclear.

We therefore used simulations and three large, independent and diverse $E$. coli sequencing datasets to investigate the robustness and reproducibility of four widelyused WGS-based AMR genotyping methods (ABRicate, ARIBA, KmerResistance, and SRST2) at scale, investigating any encountered discrepancies.

\section{Methods}

\section{AMR gene identification methods}

We evaluated the impact of different bioinformatics tools using the same AMR gene catalogue, namely the ResFinder database (v.29/10/2019). At the time the study was designed (March 2018), to be included bioinformatics tools had to: (i) have publicly available code, (ii) run on local computing architecture without major modification, (iii) accept different AMR gene databases to ensure broad and long-term typing usability, and (iv) have a command line interface that could enable batch processing of large numbers of samples (Table S1).

We identified four publicly available bioinformatic tools that met these criteria and used distinct AMR gene identification approaches: ABRicate(14) (which searches for AMR genes in assemblies using BLASTn), SRST2(7) (which maps reads directly onto the formatted AMR gene database using Bowtie 2), ARIBA(6) (which combines 
136 these two approaches, first mapping reads to the AMR gene database using minimap, and then creating local assemblies of the mapped reads using Fermi-lite) and KmerResistance(8) (which analyses shared k-mers between the query sequences and reference sequences in the AMR gene database) (Fig.S1). To mimic broad usability, each program was run using default parameters. For ABRicate, assemblies were first produced using SPAdes(15) run with default parameters.

Simulated data: single and multiple allele identification, and low coverage scenarios Prior to evaluating real data, we considered the accuracy of each method in identifying known AMR gene alleles "inserted" into simulated flanking sequence constructs. For this, each AMR gene variant in the ResFinder database $(n=3,092)$ was flanked by $1 \mathrm{~kb}$ of random sequence (using Numpy v1.16.4(16) and combined using BioPython(17) v1.74) and reads simulated at 40x coverage using ART (details and rationale in Supplementary Methods, Fig.1, S2). Other ART parameters were: error profile="HISEQ2500", mean DNA fragment length (standard deviation)=480bp (150bp), and read length $=151 \mathrm{bp}$. Each bioinformatic method was then tested to see if it could correctly identify the AMR gene variant, using default parameters.

We also considered two a priori scenarios that are thought to affect AMR genotyping(18), namely a multiple allele scenario in which multiple closely genetically related alleles (see below) of a given AMR gene were present, and a low quality scenario reflected by low sequencing coverage. For the multiple allele scenario we excluded target AMR gene variants that were incorrectly identified individually by any method (see Results), and then calculated pairwise nucleotide similarity between all remaining AMR gene variants. To do this, each remaining AMR gene variant was split into 31-mers, which were then compared with 31-mer sets from every other non-excluded AMR gene variant using pairwise Jaccard's similarity indices. AMR gene variant pairs were defined as similar if they shared any 31-mer, resulting in a total of 46,279 possible similar AMR gene variant pairs (Fig.S3-S5).

For the low coverage scenario, reads were simulated from 176 bla TEM genecontaining constructs at coverage depths ranging from $1 x$ to $50 x$ using ART ( $n=1766^{\star} 50=8,800$ simulations), reflecting total bla TEM diversity present in the ResFinder database at the time of simulation. Each construct contained a random perfect reference bla produced using Numpy/BioPython as above. Simulated reads were then processed by each genotyping method using default settings and the identified variants were compared with the known bla TEM variants present in each construct. The measure of performance for this scenario was the proportion of bla TEM variants correctly identified by each method at each coverage level.

\section{Real data: Isolate selection}

To evaluate performance on real data, we then studied a total of 1,818 E. coli isolates comprising three different WGS datasets in order to reflect different strainlevel and AMR gene diversity: (i) 984 sequentially collected bloodstream infection isolates at Oxford University Hospitals (OUH) NHS Foundation Trust(19) ("Oxford dataset"); (ii) 497 animal commensal $E$. coli isolates donated by the UK Animal and Plant Health Agency (APHA)(20) ("APHA dataset"), and (iii) 337 E. coli isolates 
185 Healthcare Associated Infections (AMRHAI) Reference Unit, which investigates isolates enriched for rare or important resistance genotypes encountered in the UK (sequenced for this study, "UKHSA dataset").

Isolates were re-cultured from frozen stocks stored in nutrient broth plus $10 \%$ glycerol at $-80^{\circ} \mathrm{C}$. DNA was extracted using the QuickGene DNA Tissue Kit S (Kurabo Industries, Japan) as per manufacturer's instructions, with an additional mechanical lysis step (FastPrep, MP Biomedicals, USA) immediately following chemical lysis. A combination of standard Illumina and in-house protocols were used to produce multiplexed paired-end libraries, which were sequenced on an Illumina HiSeq 2500 , generating $151 \mathrm{bp}$ paired-end reads. High quality sequences were denovo assembled using Velvet(21) as previously described(22). In silico Achtman(23) multi-locus sequence types (MLST) types were defined using ARIBA(6).

While this work does not attempt to predict resistance from WGS data, each isolate had linked AST (summarized in Table S2, Fig.S6), which we have included as the complexity of resistance genotype identification is associated with the phenotype. Isolates had complete AST data available for: ampicillin, ceftazidime and one other 3rd generation cephalosporin (cefotaxime for the animal commensal isolates, ceftriaxone for all others), gentamicin, ciprofloxacin, and co-trimoxazole.

We compared AMR genotypes reported for each isolate by each method, stratified by antibiotic class to which resistance was conferred as specified in the ResFinder database, namely: beta-lactams, aminoglycosides, quinolones, trimethoprim, and sulphonamides. Discrepancies were classified according to which of the four bioinformatics methods agreed (Fig.S7). The cause of discrepancy was investigated for all beta-lactam resistance genotypes, because these antibiotics are most commonly used for clinical $E$. coli infections, and then for discrepancy patterns occurring in $>1.5 \%(n=27)$ of isolates for the other classes.

\section{6. Results}

\section{Simulated scenarios}

216 Accurate identification of single AMR gene variants in simulated sequence 217 constructs

218 For the 3,092 AMR gene variants in the ResFinder database, all four genotyping 219 methods correctly identified those inserted into random sequence contexts in 2,011 $(63.5 \%)$ cases. KmerResistance was correct for all 3,092 simulations, ABRicate for 3,082 (99.7\%), ARIBA for 2,927 (94.7\%) and SRST2 for 2,120 (68.6\%) (Fig.2). For SRST2, most errors were due to its approach of pre-clustering reference sequences into sub-families by sequence identity prior to genotyping, thereby essentially excluding a priori the possibility of identifying alleles that were not selected as the representative for these sub-family clusters. This error is explained in more detail below as it also affected genotyping in real isolate sequences.

Impact of the presence of multiple closely related alleles on genotyping calls The multiple allele simulation caused significant problems for assembly-based algorithms, with ABRicate reporting fragmented/incomplete alleles for 32,194/46,279 $(70 \%)$ simulations and ARIBA reporting no alleles meeting its assembly quality requirements for $32,987 / 46,279(71 \%)$ simulations. SRST2, as expected, found only 
233 a single allele in most (33077/46,279 (71\%)) cases (Table 1), as dictated by its

234 clustering parameters. ABRicate managed to identify both alleles correctly in the absence of incorrect calls in 11,382/46,279 (25\%) of simulations, whereas ARIBA and SRST2 only managed to correctly reconstruct both members of the pair in the absence of correct calls in 2,494/46,279 (5\%) and 2,539/46,279 (5\%) cases respectively (Table 1 ). Of the four programs, KmerResistance performed the best, identifying both alleles correctly without additional erroneous calls in 38,826/46,279 $(84 \%)$. of cases. Unsurprisingly all four programs were most likely to make erroneous genotyping calls as the simulated pairs of alleles became more closely related (Fig.S8).

\section{Impact of sequencing depth on genotyping calls}

KmerResistance was able to identify bla other methods (Fig.1). Above 15x depth of coverage for the gene, all methods correctly identified bla TEM alleles in simulated constructs in $>95 \%$ of cases (Fig.1). All methods were able to identify all of the blaTEM alleles correctly at least once, but examples existed for all methods where the allele was correctly identified at low coverage, but then mis-classified at higher coverage. In general, ABRicate and SRST2, while requiring greater sequencing depth to correctly identify bla initially were more accurate at higher coverage depths, making erroneous calls for only $1 / 176(0.6 \%)$ and $0 / 176(0 \%)$ of bla TEM alleles at depths $>20 x$. In contrast, for $>20 x$ coverage ARIBA and KmerResistance made erroneous allele calls for 23/176 $(13 \%)$ and $6 / 176(3 \%)$ bla TEM variants respectively. Above 40x coverage ABRicate was incorrect for one $(0.6 \%)$, ARIBA for four $(2 \%)$, KmerResistance for one $(0.6 \%)$, and SRST2 for zero (0\%) simulated bla TEM alleles.

\section{Real data}

E. coli isolate diversity, antimicrobial susceptibility phenotypes and antimicrobial resistance genotypes

The 1,818 isolates were diverse, representing >260 multi-locus sequence types (STs), which were differentially distributed among the datasets. For example, although ST131 was the most common (207/1818 (11\%) isolates), this was largely due to the fact it was by far the most common in the UKHSA dataset $(74 / 337(22 \%)$ isolates). In the Oxford dataset, it was only the second most common ST (123/984 $(13 \%)$ isolates) after ST73 (161/984 (16\%)) isolates) and it was rare in the APHA isolates $(10 / 497$ isolates $(2 \%))$.

Correspondingly, the set also contained a broad range of resistance genes, but the exact number was dependant on the method of search. For legibility, we have included results as reported by ABRicate as this is the most conceptually simple and interrogatable approach.. The commonest AMR-associated sequence identified was $m d f A$. This is known to be universal in $E$. coli, and correspondingly was identified in all 1,818 isolates in the dataset. There were no other ubiquitous AMR genes; however, several were common across datasets, with bla $\mathrm{TEM}_{\mathrm{T}}$, aadA, sul, tet, and $d f r$ genes occurring in $>40 \%$ of the isolates. As expected, more UKHSA isolates contained extended-spectrum beta-lactamase (54/337 vs 94/1481) and carbapenemase (18/337 vs 1/1481) genes $(p=<0.001)$. Aside from blaTEM, other beta-lactamases were rare among the APHA dataset. Outside of beta-lactam- 
associated AMR genes, the Oxford dataset had the lowest proportion of other AMR genes for all the different gene families encountered in this study.

\section{Genotyping discrepancies}

10,487 different genes $(\mathrm{N}=15,588$ different alleles) were identified in the 1818 isolates by the four methods. $1,392 / 1,818(76 \%)$ isolates had discrepancies across the four bioinformatics methods for at least one gene. At the gene-level, aside from for tet, aad $A$ and cat genes, the performance of the bioinformatic tools was similar (Fig.3, panel a), with tools reporting each gene in the approximately same proportion of isolates (within $+/-2 \%$ ). With regards to the three outliers, ABRicate reported tet and aadA genes in $19 \%$ and $10 \%$ more isolates respectively than the other three tools, and ABRicate and KmerResistance reported cat genes in 5\% more isolates than ARIBA and SRST2. By contrast, the alleles reported by each tool were often discrepant, with alleles of some genes (e.g. blaSHV, blaCMY) consistently being differentially reported (Fig.3, panel b). Consequently, pairwise agreement between any two different tools was less than $59 \%(\mathrm{~N}=1,065$ isolates, Fig.3, panel c). While unsupported genotype reports (i.e. where the output of one tool was not supported by any other) were common for all tools (Fig.4), KmerResistance reported fewer unsupported genotypes than the other three tools $(p<0.001)$.

\section{Causes of genotyping discrepancy}

At least 2,530/4,321 (59\%) of allele-level discrepancies were due to programs naming the same underlying sequence differently (annotation differences). We identified three major causes of differences through investigation of discrepantly reported genes: (i) difficulty distinguishing between optimal matches among alleles with nested sequences ( $N=1,737$ genes); (ii) spurious identification of additional alleles due to reads being multiply mapped to distant variants of the same allelic family ( $\mathrm{N}=547$ genes); and (iii) tools choosing different optimal matches based on DNA sequence alignment when the database only contains one sequence per protein ( $\mathrm{N}=197)$ (Fig.5). These issues occurred alone in 1,944/2,530 (77\%) discrepantly reported genes, and or in combination in 586/2,530 (23\%) cases. In isolation these errors typically caused only a single method to be discordant, but when combined resulted in more complex patterns of discrepancy and could make all four methods disagree with one another. In addition to annotation, ABRicate's more relaxed requirement for complete gene coverage (which aims to mitigate assembly errors) caused at least 1,430/4,321 (33\%) allele-level discrepancies. Discrepancies less easily classified as (but likely related to) annotation/cut-offs did occur, but only affected 381/10487 (4\%) of reported genotypes.

\section{Annotation-related discrepancies}

The most common type of annotation error $(\mathrm{N}=1,737$ genes) was the result of tools struggling to choose optimal matches where the database contained nested sequences. One such example of this $(\mathrm{N}=24)$ was caused by the sequences for two different dfrA7 alleles in the October 2019 Resfinder database, dfrA7_1_AB161450 and dfrA7_5_AJ419170. The shorter of the two (dfrA7_1_AB161450, $4 \overline{74}$ base pairs long) aligns almost perfectly (percentage identity $=99 \%, 1$ single nucleotide gap) with the first 473 bases of dfrA7_5_AJ419170. ARIBA, KmerResistance and SRST2, which look for the best identity sequence matches, all report the sample contains a perfect match for dfrA7_1_AB161450. By contrast ABRicate, which uses BLAST to 
330 identify optimal sequences, reports the sample contains a near perfect match to

331 dfrA7_5_AJ419170, as with this being a longer match it is more statistically

332 significant. Similar errors occurred for several other genes, including sul, tet, aph(6),

333 and $\operatorname{aac}(3)$.

The second most common annotation discrepancy ( $\mathrm{N}=547$ genes) represented tools reporting multiple alleles due to reads mapping to two or more distant variants of the same allelic family. An example observed was ARIBA and SRST2 reporting multiple bla $a_{S H V}$ alleles. In this instance, ARIBA and SRST2 identified a primary perfect allele and a second allele with a lower quality match. These multiple matches however were likely spurious, with $<10$ reads mapping individually to each allele, no clear heterozygosity observed in read pileups, and no fragmentation in assembly graphs. This is the result of a biproduct of how mapping methods identify optimal matches. Both ARIBA and SRST2 map reads to each sequence in the database, and then compare "closely related" sequences to decide which mapping is optimal. Defining "closely related" however is not straightforward (Fig.S9). Reads mapping to more than one set of "closely related" sequences can result in tools finding multiple gene variants when the isolate only had one gene original

The final common annotation discrepancy ( $\mathrm{N}=197$ genes) was due to allele reporting based on which sequence in the database had the optimal DNA alignment with the target resistance gene. Although resistance gene nomenclature is largely based on protein sequence, but resistance gene databases mostly only catalogue one nucleotide sequence linked to an associated protein sequence. Variant alleles with synonymous mutations fail to perfectly match any element, and may have an alternate optimal DNA match. We observed this on 9 occasions where ABRicate, KmerResistance and SRST2 identified imperfect nucleotide-level matches to aph(3")-Ib_2_AF024602 and ARIBA identified an imperfect match to aph(3")Ib_4_AF313472. However, the sequence they were matching to in the SPAdes and A $\bar{R}$ IBA assembly was a $100 \%$ identity and coverage protein match to aph(3")Ib_5_AF321551.

\section{Non-annotation related discrepancies}

In addition to annotation discrepancies that were caused by bioinformatics algorithms, genotyping calls were also affected by partial/low coverage of AMR gene targets and assembly fragmentation, consistent with the results from simulations. For some of these, such as the 1,430 cut-off related discrepancies occurring for tet, $m f s$, $\operatorname{aad} A$, and cat genes, each program identified the same section of sequence, making it clear that the different programs had different thresholds for reporting, other situations were less clear. To investigate this in detail, we examined beta-lactamase matches which were either partial/low coverage or occurred across fragmented assemblies.

Partial/low coverage beta-lactamase genes were discrepantly found in 39 isolates

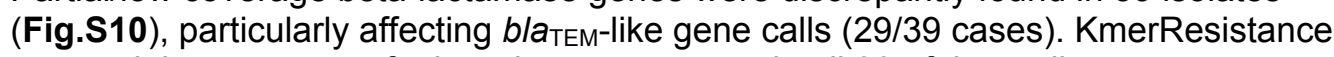
reported the presence of a beta-lactamase gene in all 39 of these discrepant cases, with calls supported to a varying degree by the other algorithms. However, in all but four cases, KmerResistance reported that the depth of the gene was less than $5 x$.

378 For the four cases where the gene was present at greater than $5 x$ depth as called by 
379 KmerResistance, three (present at depth $>100 x$ ) were omitted from ARIBA reports

380 as ARIBA assemblies contained mis-sense mutations and the final one (present at

381 depth 17x) also failed to assemble for ABRicate.

382

383

384

385

386

387

388

389

390

391

392

393

394

395

Assembly fragmentation affected ABRicate and ARIBA beta-lactam resistance gene calls in 24 cases, with 16 of these likely to be due to the presence of multiple closely related beta-lactamase alleles affecting assembly integrity. The possibility of heterozygous alleles was indicated by the ARIBA flag

"variants_suggest_collapsed_repeat", and the SRST2 "minor allele frequency value" was high ( $>20 \%$ ). KmerResistance reported two related alleles in 12/16 cases, one with high depth, percentage identity and coverage, and one much less accurately. This likely reflects KmerResistance's winner-takes-all strategy, where matching unique k-mers to reference alleles are counted, and the reference allele with the most matches is then also assigned all reads with non-unique kmer-matches. This then leaves only reads with unique k-mers matching any closely related secondary allele, resulting in poor depth and coverage metrics.

\section{Discussion}

We evaluated the impact of bioinformatics approaches to AMR genotyping in E. coli for four commonly used methods and a widely used AMR gene database (ResFinder). Using $>50,000$ simulations and comparing $>1,800$ sequences sampled across human and animal reservoirs, thereby capturing common and rare AMR genotypes, we highlight that whilst currently available, widely-used genotyping methods are useful, their outputs should be carefully considered in light of our findings. Commonly postulated causes of discrepancy, such as low quality sequencing data, appeared to play little role. Instead, discrepancies were primarily artefactual, occurring because of different approaches in representing the complexity of the reference AMR gene database. Inconsistent labelling of gene variants will also affect the reliability of any catalogue-based methods for phenotypic prediction from WGS-based AMR genotypes. Specifically, predicting phenotype based on the presence of specific allelic variants will be problematic without a reliable method of identification.

Our work agrees with previous findings by Doyle et al. on a small and selected dataset(13); however, we utilised large simulated and real-life datasets to identify these significant genotyping discrepancies between methods, and also characterized the underlying reasons for these discrepancies. We found most discrepancies were largely due to annotation differences, i.e. each method identified the same consensus sequence but then named them differently. Further, many of these discrepancies are caused by implicit and frequently incorrect assumptions about database structure and AMR gene diversity, namely: that AMR genes can be classified in well-defined families using genetic identity, that different approaches to deciding best-matching alleles are equivalent, and that isolates will usually not harbour highly genetically related variants of the same AMR gene. However, nomenclature and family structure amongst AMR genes relevant to Enterobacterales is complicated, with highly diverse genotypes (and sometimes phenotypes) being assigned similar family names (e.g. bla $a_{\mathrm{CTX}-\mathrm{M}}, b_{\mathrm{O}} \mathrm{a}_{\mathrm{OX}}$ ) and single SNPs in some cases leading to different resistance phenotypes (e.g. bla TEM-1 (Genbank: AY458016.1) - 
427 beta-lactamase inhibitor susceptible i.e. susceptible to amoxicllin-clavulanate, blaTEM-

42830 (Genbank: AJ437107.1) - beta-lactamase inhibitor resistant i.e. resistant to

429 amoxicillin-clavulanate). Given this, it is not surprising that we found methods that

430 make fewer assumptions (e.g. KmerResistance) to be more robust. Based on our

431 findings accurate resistance genotyping may require the use of multiple different

432 methods to cross-check results, and a clear understanding of the specific

433 assumptions underlying the methods used, before conclusions about allele presence

434 are drawn. The alternative is the development of new algorithms that cope better

435

436

437

438

439

440

441

442

443

444

445

446

447

448

449

450

451

452

453

454

455

456

457

458

with underlying AMR gene diversity in these organisms.

One of the key strengths of this analysis was its combined use of both simulations and real world data. By using simulations, we were able to benchmark methods against a known truth, which is impossible to do with real-world data. Previous studies using only real-world data have attempted to overcome the absence of complete knowledge of the underlying genotype by using phenotypic data as a reference standard; however genotype-phenotype correlations remain poorly defined $(10,19)$. By subsequently using a large sequencing dataset of isolates obtained across niches, we were then able to assess the extent of discrepancies in real-life, replicating the problems observed in simulated data.

A limitation of this work is that we chose not to evaluate the impact of database choice, and this will represent future work. Currently, as has been highlighted previously(24), there are discrepancies between the AMR databases in common use, with each having a slightly different scope and in some cases differential names for different AMR gene variants (e.g.strA vs aph(6)-la or $a p h D$, and strB versus $a p h(6)-I d)$. Comparing databases would have therefore added significant further complexity whilst limiting the generalisability of findings. A further limitation stemming from our fixed choice of database is that we have not analysed any methods where the bioinformatic method and database are intertwined (e.g. ResFinder/PointFinder or RGI). As the interaction between tool and database was the cause of many issues, it is possible that methods that are database-specific will perform better. However, the drawbacks of these combined resources are their inflexibility, again 459 limiting generalisability. A further limitation was that these genotyping algorithms 460 were compared using an older version of the ResFinder database - the most up to date when this work was originally planned. Since this time, 70 sequences have been added, 2 sequences modified and 2 sequences deleted (See supplementary data). We opted not to re-perform the analysis due to its manual nature and that as most of the discrepancies relate to underlying principles behind the algorithms rather than the specific implementation. Finally, we have focused our evaluation on $E$. coli, but it is likely that these issues will also more widely affect AMR genotyping, particularly of similar species with complex genotypes.

While WGS-based approaches are attractive for both characterizing AMR gene epidemiology and representing a subsequent tool for resistance prediction, this work highlights the need for caution when interpreting resistance genotypes reported by even widely used bioinformatics methods. Before WGS-based approaches can be 473 considered reliable for use in E. coli (and likely other Enterobacterales), particularly 474 for clinical decision making or replacing phenotypic data to determine 
475 epidemiological trends, database standardisation, the development of novel

476 genotyping approaches, and improved validation and evaluation will be required.

\section{Author statements}

\subsection{Authors and contributors}

480 TJD, NS, AES, ASW, DWC and TEAP conceptualised the study. TD, NS, ASW, AES

481 and MFA decided the methodology. NS, ASW, MFA, AES, DWC and TEAP

482 supervised the project. NS, MA, MFA, MJE, KH and SH acquired and curated the

483 data used in this study. TJD and JSW constructed software pipelines to analyse

484 sequencing data using each of the bioinformatic tools. TJD and ASW investigated

485 the data. TJD performed the formal analysis. NS, AES, SL, HP, AES and TEAP

486 assisted with interpreting the cause and impact of discrepancies. TJD and NS wrote

487 the original draft. TJD, NS, AES, PWF, TEAP and ASW assisted with data

488 visualisation. All authors were involved in the review and editing process.

\subsection{Conflicts of interest}

490 The authors have no conflicts of interest to declare.

\subsection{Funding information}

492 The study was funded by the National Institute for Health Research Health

493 Protection Research Unit (NIHR HPRU) in Healthcare Associated Infections and

494 Antimicrobial Resistance at Oxford University in partnership with Public Health

495 England (PHE) [NIHR200915]. DWC, TEAP, PWF and ASW are supported by the

496 NIHR Oxford Biomedical Research Centre. The report presents independent

497 research funded by the National Institute for Health Research. The views expressed

498 in this publication are those of the authors and not necessarily those of the NHS, the

499 National Institute for Health Research, the Department of Health or Public Health

500 England. NS is an Oxford Martin Fellow and an NIHR Oxford BRC Senior Fellow.

501 ASW is an NIHR Senior Investigator.

502

\section{$503 \quad 8.4$ Ethical approval}

504 Not applicable.

\subsection{Acknowledgements}

507 We are grateful to the microbiology laboratory teams at the John Radcliffe Hospital,

508 Oxford, the Animal and Plant Health Agency, and UK Health Security Agency.

\section{9. References}

510 1. Quainoo S, Coolen JPM, van Hijum S, Huynen MA, Melchers WJG, van 511 Schaik W, et al. Whole-Genome Sequencing of Bacterial Pathogens: the Future of 512 Nosocomial Outbreak Analysis. Clin Microbiol Rev. 2017;30(4):1015-63.

513 2. Quan TP, Bawa Z, Foster D, Walker T, Del Ojo Elias C, Rathod P, et al.

514 Evaluation of Whole-Genome Sequencing for Mycobacterial Species Identification 515 and Drug Susceptibility Testing in a Clinical Setting: a Large-Scale Prospective 516 Assessment of Performance against Line Probe Assays and Phenotyping. J Clin 517 Microbiol. 2018;56(2). 
518 3. Bortolaia V, Kaas RS, Ruppe E, Roberts MC, Schwarz S, Cattoir V, et al.

519 ResFinder 4.0 for predictions of phenotypes from genotypes. J Antimicrob

520 Chemother. 2020;75(12):3491-500.

521 4. Alcock BP, Raphenya AR, Lau TTY, Tsang KK, Bouchard M, Edalatmand A,

522 et al. CARD 2020: antibiotic resistome surveillance with the comprehensive antibiotic

523 resistance database. Nucleic Acids Res. 2020;48(D1):D517-d25.

5245 5. Feldgarden M, Brover V, Haft DH, Prasad AB, Slotta DJ, Tolstoy I, et al.

525 Validating the AMRFinder Tool and Resistance Gene Database by Using

526 Antimicrobial Resistance Genotype-Phenotype Correlations in a Collection of

527 Isolates. Antimicrob Agents Chemother. 2019;63(11).

528 6. Hunt M, Mather AE, Sánchez-Busó L, Page AJ, Parkhill J, Keane JA, et al.

529 ARIBA: rapid antimicrobial resistance genotyping directly from sequencing reads.

530 Microb Genom. 2017;3(10):e000131.

531 7. Inouye M, Dashnow H, Raven LA, Schultz MB, Pope BJ, Tomita T, et al.

532 SRST2: Rapid genomic surveillance for public health and hospital microbiology labs.

533 Genome medicine. 2014;6(11):90.

534 8. Clausen PT, Zankari E, Aarestrup FM, Lund O. Benchmarking of methods for 535 identification of antimicrobial resistance genes in bacterial whole genome data. J

536 Antimicrob Chemother. 2016;71(9):2484-8.

537 9. Zankari E, Allesøe R, Joensen KG, Cavaco LM, Lund O, Aarestrup FM.

538 PointFinder: a novel web tool for WGS-based detection of antimicrobial resistance

539 associated with chromosomal point mutations in bacterial pathogens. J Antimicrob

540 Chemother. 2017;72(10):2764-8.

541 10. Stoesser N, Batty EM, Eyre DW, Morgan M, Wyllie DH, Del Ojo Elias C, et al.

542 Predicting antimicrobial susceptibilities for Escherichia coli and Klebsiella

543 pneumoniae isolates using whole genomic sequence data. J Antimicrob Chemother.

544 2013;68(10):2234-44.

545 11. Shelburne SA, Kim J, Munita JM, Sahasrabhojane P, Shields RK, Press EG,

546 et al. Whole-Genome Sequencing Accurately Identifies Resistance to Extended-

547 Spectrum $\beta$-Lactams for Major Gram-Negative Bacterial Pathogens. Clin Infect Dis.

548 2017;65(5):738-45.

549 12. Stubberfield E, AbuOun M, Sayers E, O'Connor HM, Card RM, Anjum MF.

550 Use of whole genome sequencing of commensal Escherichia coli in pigs for

551 antimicrobial resistance surveillance, United Kingdom, 2018. Euro Surveill.

552 2019;24(50).

553 13. Doyle RM, O'Sullivan DM, Aller SD, Bruchmann S, Clark T, Coello Pelegrin A,

554 et al. Discordant bioinformatic predictions of antimicrobial resistance from whole-

555 genome sequencing data of bacterial isolates: an inter-laboratory study. Microb

556 Genom. 2020;6(2).

557 14. Seemann T. ABRicate. 2020.

558 15. Bankevich A, Nurk S, Antipov D, Gurevich AA, Dvorkin M, Kulikov AS, et al.

559 SPAdes: a new genome assembly algorithm and its applications to single-cell

560 sequencing. J Comput Biol. 2012;19(5):455-77.

561 16. Harris CR, Millman KJ, van der Walt SJ, Gommers R, Virtanen P,

562 Cournapeau D, et al. Array programming with NumPy. Nature. 2020;585(7825):357-

56362.

564 17. Cock PJ, Antao T, Chang JT, Chapman BA, Cox CJ, Dalke A, et al.

565 Biopython: freely available Python tools for computational molecular biology and

566 bioinformatics. Bioinformatics. 2009;25(11):1422-3. 
567 18. Ellington MJ, Ekelund O, Aarestrup FM, Canton R, Doumith M, Giske C, et al.

568 The role of whole genome sequencing in antimicrobial susceptibility testing of

569 bacteria: report from the EUCAST Subcommittee. Clin Microbiol Infect. 2017;23(1):2-

57022.

571 19. Davies TJ, Stoesser N, Sheppard AE, Abuoun M, Fowler P, Swann J, et al.

572 Reconciling the Potentially Irreconcilable? Genotypic and Phenotypic Amoxicillin-

573 Clavulanate Resistance in Escherichia coli. Antimicrob Agents Chemother.

574 2020;64(6).

575 20. AbuOun M, O'Connor HM, Stubberfield EJ, Nunez-Garcia J, Sayers E, Crook

576 DW, et al. Characterizing Antimicrobial Resistant Escherichia coli and Associated

577 Risk Factors in a Cross-Sectional Study of Pig Farms in Great Britain. Front

578 Microbiol. 2020;11:861.

579 21. Zerbino DR. Using the Velvet de novo assembler for short-read sequencing

580 technologies. Curr Protoc Bioinformatics. 2010; Chapter 11:Unit 115.

581 22. Stoesser N, Sheppard AE, Peirano G, Anson LW, Pankhurst L, Sebra R, et al.

582 Genomic epidemiology of global Klebsiella pneumoniae carbapenemase (KPC)-

583 producing Escherichia coli. Sci Rep. 2017;7(1):5917.

584 23. Wirth T, Falush D, Lan R, Colles F, Mensa P, Wieler LH, et al. Sex and

585 virulence in Escherichia coli: an evolutionary perspective. Mol Microbiol.

586 2006;60(5):1136-51.

587 24. McArthur AG, Tsang KK. Antimicrobial resistance surveillance in the genomic 588 age. Ann N Y Acad Sci. 2017;1388(1):78-91. 
bioRxiv preprint doi: https://doi.org/10.1101/2021.11.03.467004; this version posted November 3, 2021. The copyright holder for this preprint (which was not certified by peer review) is the author/funder, who has granted bioRxiv a license to display the preprint in perpetuity. It is made available under aCC-BY 4.0 International license.

589 10. Figures and tables

590 Figure 1. Proportion of correct genotype calls for single AMR gene variants in 591 simulated constructs by coverage depth and bioinformatics method.

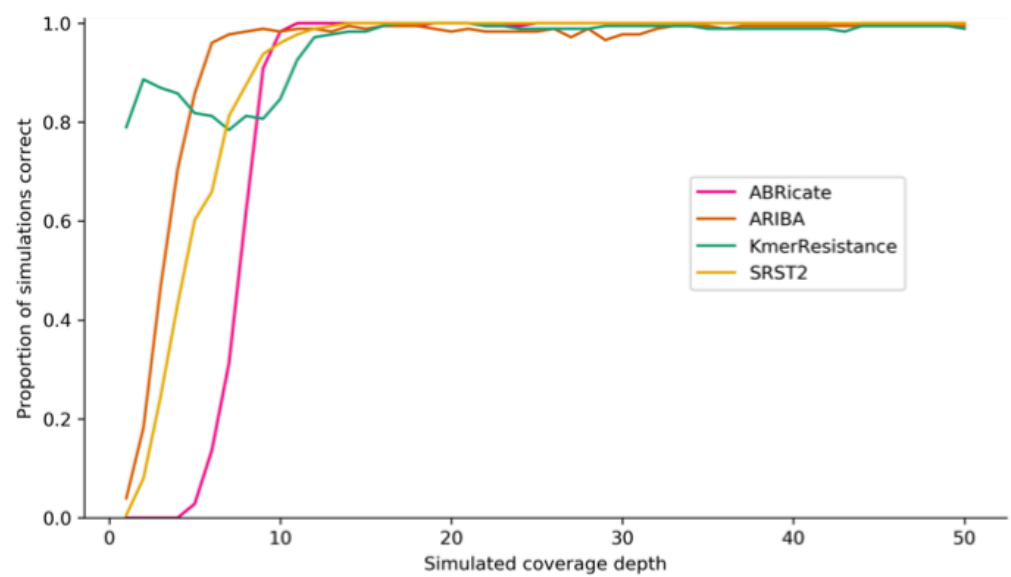


bioRxiv preprint doi: https://doi.org/10.1101/2021.11.03.467004; this version posted November 3, 2021. The copyright holder for this preprint (which was not certified by peer review) is the author/funder, who has granted bioRxiv a license to display the preprint in perpetuity. It is made available under aCC-BY 4.0 International license.

593 Figure 2. Identification of known single AMR gene variants in simulated

594 contexts by bioinformatic method. Note only cases where one or more methods 595 were incorrect are shown $(n=1,081)$. "+" denotes the case where total SRST2-only

596 errors $=906$, but are truncated to 200 to make other errors visible. blue $=$ method

597 correct for these simulations, orange $=$ method incorrect.

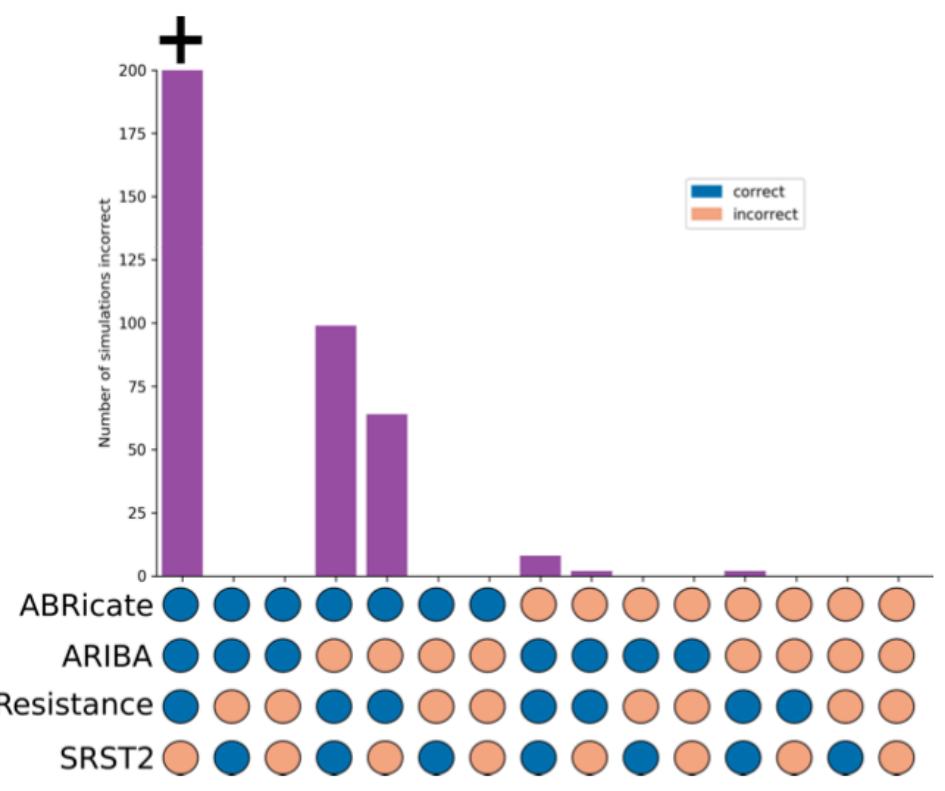


bioRxiv preprint doi: https://doi.org/10.1101/2021.11.03.467004 this version posted November 3, 2021. The copyright holder for this preprint (which was not certified by peer review) is the author/funder, who has granted bioRxiv a license to display the preprint in perpetuity. It is made available under aCC-BY 4.0 International license.

602

603

604

605

606

607

608

609

610

Figure 3. Gene identification concordance vs allele identification concordance. a) The number of isolates containing at least one allele of the name gene families ( $x$ axis) stratified by method. b) The proportion of times a given gene was identified concordantly by all four methods. c) Pairwise agreement between the different methods across all isolates.

a)

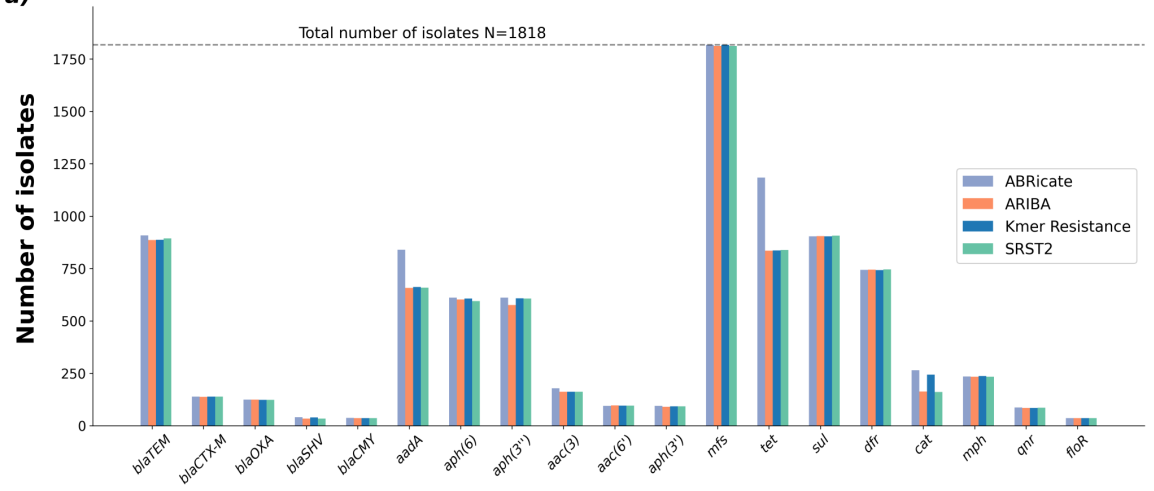

b)

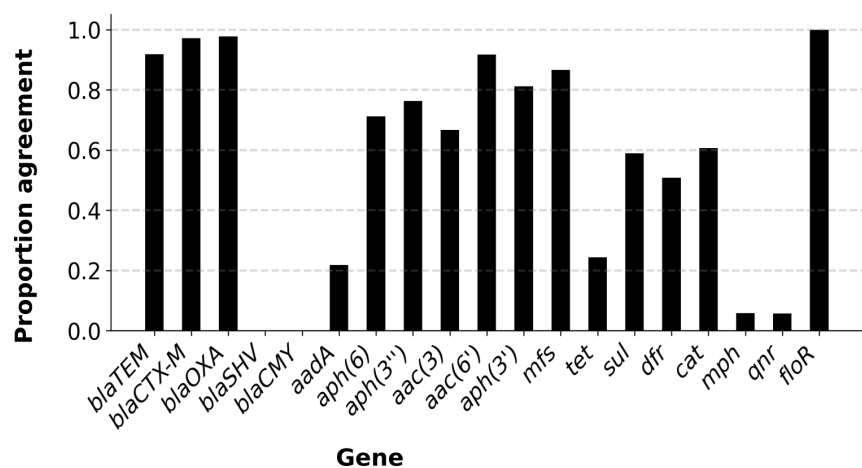

c)

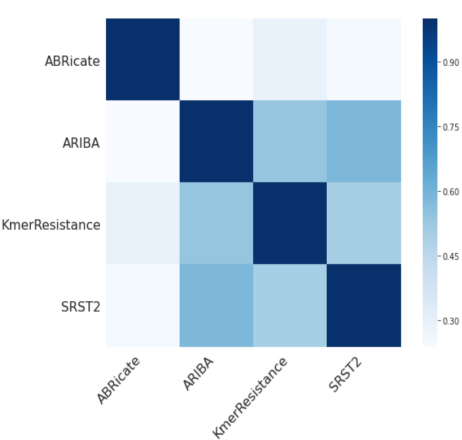


bioRxiv preprint doi: https://doi.org/10.1101/2021.11.03.467004; this version posted November 3, 2021. The copyright holder for this preprint (which was not certified by peer review) is the author/funder, who has granted bioRxiv a license to display the preprint in perpetuity. It is made available under aCC-BY 4.0 International license.

611 Figure 4. Genotype calls produced by a single method only, stratified by 612 antibiotic class.

614

613

614

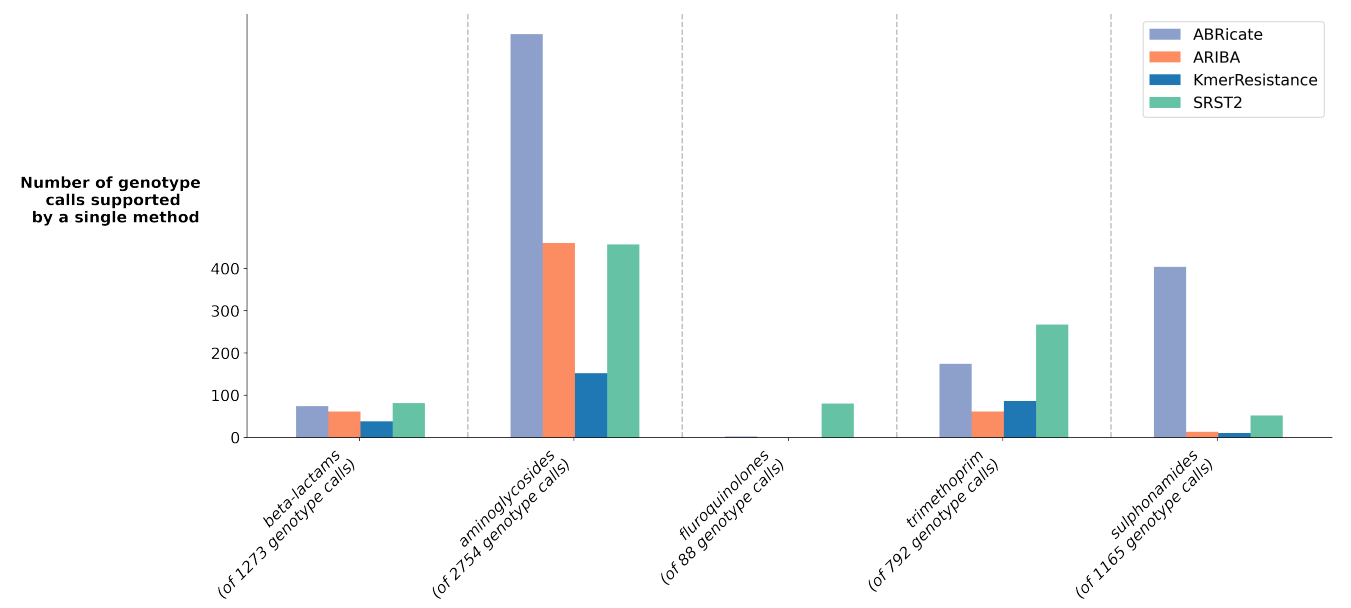

616 
bioRxiv preprint doi: https://doi.org/10.1101/2021.11.03.467004; this version posted November 3, 2021. The copyright holder for this

preprint (which was not certified by peer review) is the author/funder, who has granted bioRxiv a license to display the preprint in perpetuity. It is made available under aCC-BY 4.0 International license.

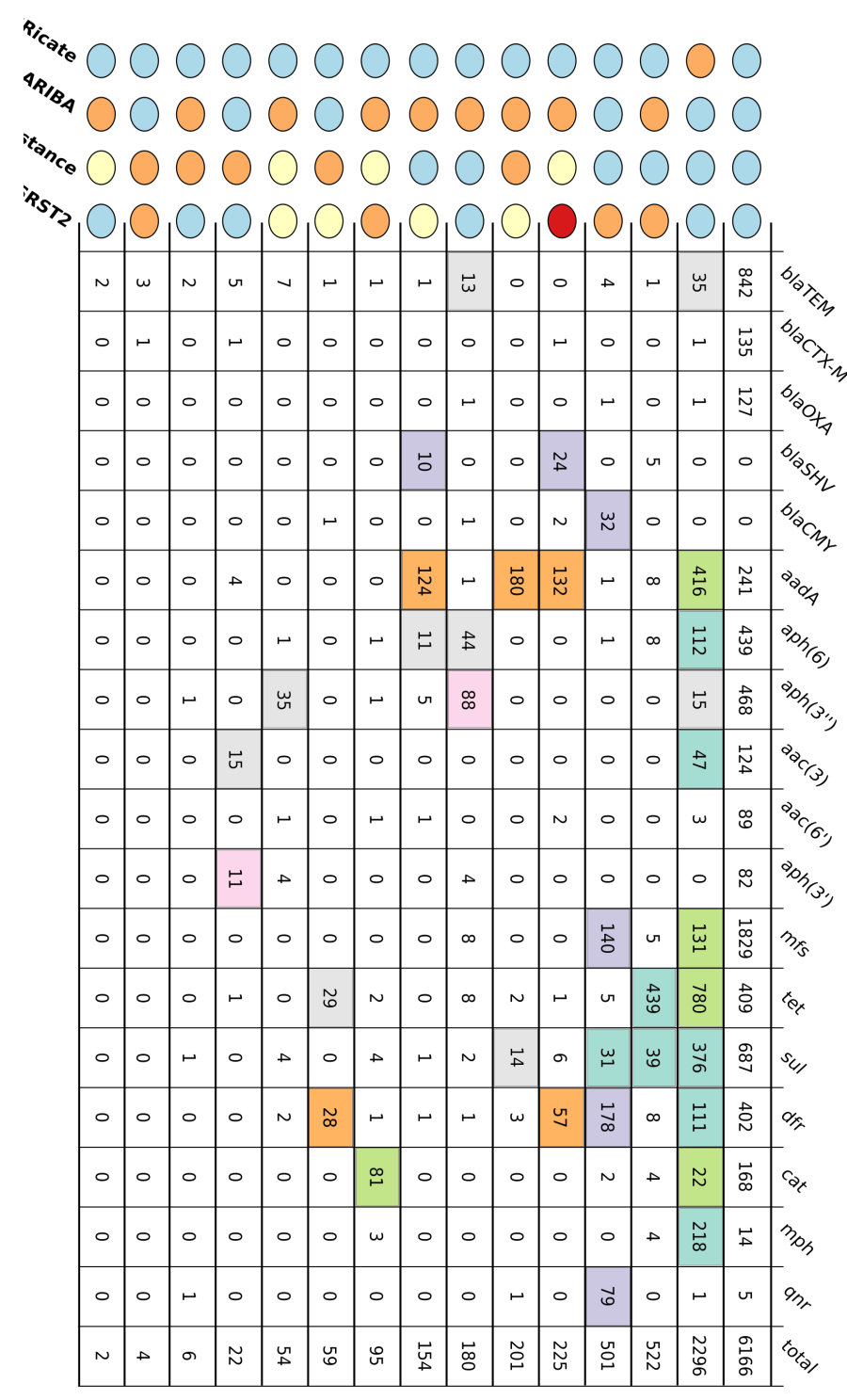

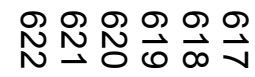

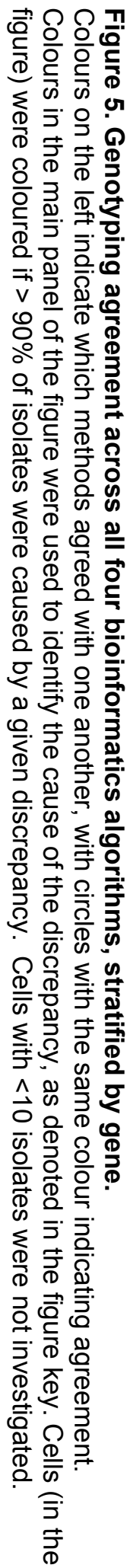


624 Table 1. Performance of genotyping methods in evaluating simulated

625 constructs with two related allelic variants. Percentage reported out of a total of 62646,279 simulations performed for each method.

\begin{tabular}{|l|l|l|l|l|}
\hline \multicolumn{2}{|l|}{ Number of calls (\%) } \\
\hline $\begin{array}{l}\text { Genotyping } \\
\text { call }\end{array}$ & ABRicate & ARIBA & KmerResistance & SRST2 \\
\hline $\begin{array}{l}\text { No correct } \\
\text { calls }\end{array}$ & $17,145(37 \%)$ & $36,150(78 \%)$ & $489(1 \%)$ & $9,898(21 \%)$ \\
\hline $\begin{array}{l}\text { One correct } \\
\text { call but } \\
\text { additional } \\
\text { incorrect calls }\end{array}$ & $2,419(5 \%)$ & $2(0 \%)$ & $1,452(3 \%)$ & $152(0 \%)$ \\
\hline $\begin{array}{l}\text { One correct } \\
\text { call, no } \\
\text { incorrect calls }\end{array}$ & $15,333(33 \%)$ & $7,634(17 \%)$ & $2,203(5 \%)$ & $33,077(71 \%)$ \\
\hline $\begin{array}{l}\text { Two correct } \\
\text { calls, but } \\
\text { additional } \\
\text { incorrect calls }\end{array}$ & $0(0 \%)$ & $1(0 \%)$ & $3,309(7 \%)$ & $613(1 \%)$ \\
\hline $\begin{array}{l}\text { Two correct } \\
\text { calls, no } \\
\text { incorrect calls }\end{array}$ & $11,382(25 \%)$ & $2494(5 \%)$ & $33826(84 \%)$ & $2539(5 \%)$ \\
\hline
\end{tabular}

